\title{
ON COMPLEXITY AND MOTION PLANNING FOR CO-RANK ONE SUB-RIEMANNIAN METRICS
}

\author{
Cutberto Romero-Meléndez ${ }^{1}$, Jean Paul Gauthier ${ }^{2}$ And \\ FELIPE MONROY-PÉREZ ${ }^{3}$
}

\begin{abstract}
In this paper, we study the motion planning problem for generic sub-Riemannian metrics of co-rank one. We give explicit expressions for the metric complexity (in the sense of Jean [10,11]), in terms of the elementary invariants of the problem. We construct asymptotic optimal syntheses. It turns out that among the results we show, the most complicated case is the 3-dimensional. Besides the generic $C^{\infty}$ case, we study some non-generic generalizations in the analytic case.
\end{abstract}

Mathematics Subject Classification. 34H05, 49J15, 53C17.

Received July 7, 2003. Revised February 26, 2004.

\section{Introduction, NOTATIONS AND ORGANIZATION OF THE PAPER}

The motion planning problem has been extensively studied in the literature. The problem is relevant both theoretically and in applications in areas such as robotics and constructive controllability. A fair bibliographical recount of the problem goes beyond the limits of this paper, we limit ourselves referring the reader to the paper [15] and the book [7], together with all the references therein.

Roughly speaking, the problem has three elements, a smooth manifold $M$, a control system $\Psi$ on $M$ and a pair of points $m_{i}, m_{f} \in M$. The motion planning problem then consists of finding a $\Psi$-admissible trajectory $c:\left[0, T_{c}\right] \rightarrow M$, satisfying $c(0)=m_{i}$ and $c\left(T_{c}\right)=m_{f}$, with further requirements (avoiding certain obstacles, .. ) and with "low cost". In general, this is achieved in two steps.

One starts by constructing a non-admissible trajectory $\Gamma$ connecting $m_{i}, m_{f}$ (and avoiding the obstacles). This is a purely geometric problem and we will not consider it here.

The second step consists of approximating $\Gamma$ by a $\Psi$-admissible trajectory $\gamma$ (with low cost if possible). The complexity of the approximation then enters into the picture.

The precise statements and the rigorous convergence theorems are very involved and appear interspersed in the literature under different approaches. For deriving our results on complexity and motion planning problem, we adopt the sub-Riemannian geometry view point which, on one hand, possesses enough generality to handle the non-holonomic motion planning problem for controllable systems without drift, and on the other hand,

\footnotetext{
Keywords and phrases. Motion planning problem, metric complexity, normal forms, asymptotic optimal synthesis.

1 Laboratoire d'Analyse Appliquée et Optimisation, Département de Mathématiques, Université de Bourgogne, 21078 Dijon, France.

2 Departement Maths, Lab. LE2I, UMR CNRS 5158, Université de Bourgogne, BP 47870, 21078 Dijon, France.

3 Basic Sciences Department, UAM-Azcapotzalco, 02200, México D.F., Mexico; e-mail: fmp@correo.azc.uam.mx
} 
enables us to use strong geometric techniques, such as normal forms and geometric invariants. Furthermore, we restrict ourselves to co-rank one sub-Riemannian metrics.

\subsection{Definitions}

Through all this paper, $\Xi_{N}$ denotes a manifold of dimension $N$ with $N \geq 3$, everything is smooth, which means at least $C^{\infty}$ and for technical reasons $C^{\omega}$ when explicitly stated. A sub-Riemannian metric over $\Xi_{N}$ is a pair $(\Delta, g)$, where $\Delta$ is a distribution over $\Xi_{N}$ and $g$ is a Riemannian metric over $\Delta$. An admissible curve is an absolutely continuous curve $\sigma:\left[0, T_{\sigma}\right] \rightarrow \Xi_{N}$, such that $\dot{\sigma}(t) \in \Delta(\sigma(t))$ a.e., the sub-Riemannian length of such a $\sigma(t)$ is defined as follows

$$
\ell(\sigma)=\int_{0}^{T_{\sigma}} \sqrt{g(\dot{\sigma}(\tau), \dot{\sigma}(\tau))} \mathrm{d} \tau
$$

and the sub-Riemannian distance between two arbitrary points $p, q \in \Xi_{N}$ is defined as

$$
\mathrm{d}(p, q)=\inf \left\{\ell(\sigma) \mid \sigma:\left[0, T_{\sigma}\right] \rightarrow \Xi_{N}, \text { is admissible and } \sigma(0)=p, \sigma\left(T_{\sigma}\right)=q\right\} .
$$

Sub-Riemannian geometry merges subtle differential geometric issues, together with a novel approach for tackling problems in non-linear geometric control. We limit our discussion to the metric complexity associated to a subRiemannian metric $(\Delta, g)$ where $\Delta$ is a co-rank 1 distribution. For a more general treatment of sub-Riemannian geometry we refer the reader to the survey [14].

Definition 1.1. A motion planning problem on $\Xi_{N}, N \geq 3$ is a triple $\Sigma=(\Delta, g, \Gamma)$, where $(\Delta, g)$ is a sub-Riemannian metric over $\Xi_{N}$, and $\Gamma:[0,1] \rightarrow \Xi_{N}$ is a smooth parameterized curve.

We shall consider generic motion planning problems, the set of all motion planning problems endowed with the $C^{\infty}$ topology is denoted by $S^{\infty}$. In the analytic case, we use the notation $S^{\omega}$ and we consider the trace on $S^{\omega}$ of the $C^{\infty}$ topology. Since $\Gamma$ is compact and since the problem depends only on the germ of $(\Delta, g)$ along $\Gamma$, there is no need to consider the $C^{\infty}$ Whitney topology.

Definition 1.2. A motion planning problem $\Sigma=(\Delta, g, \Gamma)$, is said to be relevant if $\Gamma(t)$ is transversal to $\Delta(\Gamma(t))$ for all $t \in[0,1]$.

We denote by $S_{R}^{\infty}$ and $S_{R}^{\omega}$ the corresponding sets of relevant systems, they form open subsets of $S^{\infty}$ and $S^{\omega}$ respectively.

In order to study the metric complexity associated to a motion planning problem $\Sigma$, we follow Jean's definition, see [10] and [11], we start by taking a small real parameter $\varepsilon \geq 0$ and by denoting by $T_{\varepsilon}$ the sub-Riemannian tube centered on $\Gamma$ with radius $\varepsilon$, that is $T_{\varepsilon}=\left\{x \in \Xi_{N} \mid \mathrm{d}(x, \Gamma) \leq \varepsilon\right\}$.

Definition 1.3. The metric complexity of $\Sigma$ is defined as $M C_{\Sigma}(\varepsilon)=\frac{1}{\varepsilon} \min _{\gamma}\{\operatorname{SR}$-length $(\gamma)\}$, where the minimum is taken among all admissible curves $\gamma:\left[0, T_{\gamma}\right] \rightarrow \Xi_{N}$, entirely contained in the tube $T_{\varepsilon}$ and joining $\Gamma(0)$ to $\Gamma(1)$, that is, $\gamma(0)=\Gamma(0)$ and $\gamma\left(T_{\gamma}\right)=\Gamma(1)$.

Since we are interested in the asymptotic behavior of the metric complexity when $\varepsilon \rightarrow 0$, it is convenient to consider asymptotic equivalents. Although this is very elementary, to be perfectly clear, let us recall precisely which kind of asymptotic equivalent we consider.

Definition 1.4. Two functions $\varphi_{1}(\varepsilon)$ and $\varphi_{2}(\varepsilon)$ tending to $\infty$ when $\varepsilon \rightarrow 0$ are said weakly equivalent (denoted $\varphi_{1} \stackrel{w}{\simeq} \varphi_{2}$ ), if $\frac{\varphi_{2}(\varepsilon)}{\varphi_{1}(\varepsilon)}$ and $\frac{\varphi_{1}(\varepsilon)}{\varphi_{2}(\varepsilon)}$, are bounded when $\varepsilon \rightarrow 0$. They are said strongly equivalent (denoted $\varphi_{1} \stackrel{s}{\simeq} \varphi_{2}$ ), if $\lim _{\varepsilon \rightarrow 0} \frac{\varphi_{1}(\varepsilon)}{\varphi_{2}(\varepsilon)}=1$.

In this paper, we shall take both, the metric complexity and its (weak or strong) equivalent, indistinctly. Contrarily to the results of Jean [10-12], most of our expressions for the metric complexity in this paper, are in the sense of strong equivalence. 


\subsection{The fundamental 2 -form}

Let $\omega$ be a one-form such that $\Delta=\operatorname{ker} \omega$. We can choose it in such a way that $\omega\left(\frac{\mathrm{d} \Gamma}{\mathrm{d} t}(t)\right)=1$, for all $t \in[0,1]$. This one-form $\omega$ is defined modulo multiplication by a smooth function $f$ that takes value 1 on $\Gamma$.

We take $\alpha=\mathrm{d} \omega$ and consider $\tilde{\alpha}=\left.\alpha\right|_{\Delta}$. The map $t \mapsto \widetilde{\alpha}(\Gamma(t))$ determines along $\Gamma$ a field of 2 -forms on $\Delta$. This field is well defined and it is independent of $f$. In fact, since

$$
\widetilde{\alpha}=\left.\mathrm{d}(f \omega)\right|_{\Delta}
$$

then for all $G, H$ sections of $\Delta$, we have

$$
\widetilde{\alpha}(G, H)(\Gamma(t))=\mathrm{d}(f \omega)(G, H)=\mathrm{d} f \wedge \omega(G, H)+f \mathrm{~d} \omega(G, H)=0+f \mathrm{~d} \omega(G, H)
$$

but $f(\Gamma(t))=1$, then $\widetilde{\alpha}(\Gamma(t))$ is independent of $f$.

Definition 1.5. We call $\widetilde{\alpha}$ the fundamental 2 -form associated to $\Sigma$.

The form $\widetilde{\alpha}$ defines a skew symmetric (with respect to $g$ ) linear map $A(t): \Delta(\Gamma(t)) \longrightarrow \Delta(\Gamma(t)$ ), in the following way:

$$
g(A(t) X, Y)=\widetilde{\alpha}(X, Y), \quad \text { for } \quad X \text { and } Y \text { in } \Delta(\Gamma(t)) .
$$

Then, once an orthonormal frame $F_{t}$ is given in $\Delta(\Gamma(t))$, it defines an element of $\mathfrak{s o}(N-1)$ given by the matrix of $A(t)$ in the basis $F_{t}$. This matrix shall be typically denoted by $\bar{A}(t)$.

\subsection{Statement of our results}

Let $\kappa(t)=\sup \{\mid$ eigenvalues of $A(t) \mid\}$. Let us denote by $M C_{\Sigma}(\varepsilon, T)$ the metric complexity of the curve $\Gamma:[0, T] \rightarrow \Xi_{N}$, for $T<1$. The following theorems are our main results for the dimensions $N \geq 4$ and $N=3$.

Theorem 1.6. $(N \geq 4)$ There is an open and dense subset of $S_{R}^{\infty}$ (and also of $S^{\infty}$, but in that case $\kappa(t)$ is well defined except on a finite subset of $[0,1])$, such that

$$
M C_{\Sigma}(\varepsilon, T) \stackrel{s}{\simeq} \frac{2}{\varepsilon^{2}} \int_{0}^{T} \frac{\mathrm{d} t}{\kappa(t)}
$$

This integral is well defined and finite. Moreover, the function $T \mapsto \int_{0}^{T} \frac{\mathrm{d} t}{\kappa(t)}$ is smooth, for $\Sigma \in S_{R}^{\infty}$.

Theorem 1.7. $(N=3)$ There is an open and dense subset of $S_{R}^{\infty}$ (and also of $S^{\infty}$ ), such that

$$
M C_{\Sigma}(\varepsilon, T) \stackrel{s}{\gtrsim}-\frac{\ln \varepsilon}{\varepsilon^{2}} \sum_{i=1}^{M} C\left(t_{i}\right)
$$

where $t_{i} \in[0, T], \Gamma\left(t_{i}\right)$ is a Martinet point of $\Delta$ (i.e. $\Gamma\left(t_{i}\right)$ is a point at which $\Delta$ has a Martinet singularity, that is $\kappa\left(t_{i}\right)=0$ and $\left.\frac{\mathrm{d} \kappa}{d t}\left(t_{i}^{+}\right) \neq 0\right)$, and $C\left(t_{i}\right)$ are constants. If there are no Martinet points, then the formula for $M C_{\Sigma}(\varepsilon, T)$ is still (2) in Theorem 1.6.

In this paper we do not compute explicitly the constants $C\left(t_{i}\right)$ for the general case, but we provide an example for which the asymptotic inequality (3) becomes an asymptotic equality, together with some estimations for the constants $C\left(t_{i}\right)$.

In the analytic case but non generic, under the assumption that the fundamental form $\widetilde{\alpha}(t)$ never vanishes, we prove that the asymptotics for the metric complexity is still given by (2), more specifically we prove the 
following theorem:

Theorem 1.8. If $\widetilde{\alpha}$ is never zero, and $\Sigma \in S_{R}^{\omega}$ (or $\left.S^{\omega}\right)$, then the asymptotics for the metric complexity is given by formula (2) of Theorem 1.6. In this case, the map $T \mapsto \int_{0}^{T} \frac{\mathrm{d} t}{\kappa(t)}$ is only $C^{1}$, piecewise analytic and its second derivative has jumps at isolated points.

Definition 1.9. A weak (strong) asymptotic optimal synthesis is a control strategy, depending on $\varepsilon$, that realizes a weak (strong) equivalent of the metric complexity, when $\varepsilon \rightarrow 0$, i.e., it is a family $\left\{\gamma_{\varepsilon}\right\}$, of admissible curves, $\gamma_{\varepsilon}\left(\left[0, \theta_{\varepsilon}\right]\right) \subset T_{\varepsilon}$ satisfying $\gamma_{\varepsilon}(0)=\Gamma(0)$ and $\gamma_{\varepsilon}\left(\theta_{\varepsilon}\right)=\Gamma(1)$, such that,

$$
\frac{1}{\varepsilon}\left(\operatorname{SR} \text {-length }\left(\gamma_{\varepsilon}\right)\right) \simeq M C_{\Sigma}(\varepsilon)
$$

In this paper, we will describe explicit asymptotic optimal syntheses (strong), in the case of Theorems 1.6 and 1.8 above. We will also show a (weak) explicit optimal synthesis in the Martinet case.

Our results have to be compared to those of Jean in papers [10] and [11], on which the asymptotics $\frac{1}{\varepsilon^{2}}$ and $-\frac{\log \varepsilon}{\varepsilon}$ already appear, however, with his technique, he only obtains weak equivalence. Also, in his papers, there is no explicit construction of an asymptotic optimal synthesis. On the other hand, the weakness of our paper with respect to F. Jean's results is the fact that we consider distributions of codimension one only.

\subsection{Remarks, comments and notations}

Non-relevant motion planning problems: generically, for $\Sigma \in S^{\infty}$ or $\Sigma \in S^{\omega}$, the distribution $\Delta$ is tangent to the curve $\Gamma$ at isolated points. Around these points, since the curve $\Gamma$ is almost admissible, asymptotic optimal synthesis is rather trivial. In fact, if $t_{0}$ is such a point we have that $\lim _{t \rightarrow t_{0}} \kappa(t)=+\infty$, the integral in (2) is well defined and still yields the metric complexity.

Regularity: first we assume that the curve $\Gamma$ is regular, that is, $\frac{\mathrm{d} \Gamma}{\mathrm{d} t}(t) \neq 0$ for all $t \in[0,1]$. Second, since $N \geq 3$, it is clear that the set of motion planning problems, with $\Delta$ non integrable (otherwise it does not define a sub-Riemannian metric), and with $\Gamma$ having no double points, is an open and dense set in $S^{\infty}\left(S^{\omega}\right)$. We limit ourselves to this case, and we denote again by $S^{\infty}\left(S^{\omega}\right)$ the set of such problems.

Orthonormal frames for $g$ : we shall consider co-rank 1 distributions only, that is, the dimension of $\Delta_{x}$ is $N-1$ for all $x \in \Xi_{N}$. A convenient way to specify the corresponding sub-Riemannian metric, is by means of $N-1$ vector fields over $\Xi_{N}$ that are everywhere independent, generate the distribution $\Delta$, and provide at each point an orthonormal frame for $g$. Since we are assuming that $\Gamma$ does not have double points and since our problem depends only on the germ of the $(\Delta, g)$ along $\Gamma$, then it is always possible to find an orthonormal frame $F$ for the sub-Riemannian structure, globally defined in a neighborhood of $\Gamma$. Therefore, we can restrict ourselves to the case where $\Xi_{N}$ is a fixed open connected subset of $\mathbb{R}^{N}$, and the metric is specified by a global orthonormal frame. Therefore we allow to write $\Sigma=(F, \Gamma)$, where $F$ is a $(N-1)$-tuple of independent vector fields globally defined. If a coordinate system $(x, w)$ is fixed with $x \in \mathbb{R}^{N-1}$ and $w \in \mathbb{R}$, we will also write $\Sigma=(Q, L, \Gamma)$, where: $F=\left(F_{1}, \ldots, F_{N-1}\right)$ and

$$
F_{j}=\sum_{i=1}^{N-1} Q_{i j}(x, w) \frac{\partial}{\partial x_{i}}+L_{j}(x, w) \frac{\partial}{\partial w}, \quad j=1, \ldots, N-1 .
$$

\subsection{Organization of the paper}

Apart from this introduction the paper contains five sections and one appendix. In Section 2, we state and prove some genericity results about co-rank 1 sub-Riemannian metrics and motion-planning problems. 
In Section 3, we present normal forms and normal coordinates for motion-planning problems. These tools are the key points in our treatment. The idea of normal coordinates and the resulting normal forms were introduced for the 3-dimensional case in the papers [2,5], the general contact case is discussed in [3]. For the quasi-contact case, they were introduced in [6]. Here, we generalize these normal forms and normal coordinates to generic motion-planning problems, and also to degenerate ones, in the analytic case.

The normal coordinates are the sub-Riemannian analogs of the classical normal coordinates in Riemannian geometry. Normal forms in the Riemannian case, similar to those presented in this paper, were already well known by Darboux.

In Section 4, we use these normal forms for $N \geq 4$ (or $N=3$, but contact) to construct in a rather natural way an asymptotic optimal synthesis, then we obtain the asymptotics (2) for the metric complexity of Theorems 1.6 and 1.7 .

Section 5 is devoted to the Martinet Case. We prove Theorem 1.7, and, for an example, we show in a constructive way, the equality in the asymptotics (3), i.e., we exhibit an asymptotic optimal synthesis (but weak).

In Section 6, we consider non generic situations, but analytic. We show that our normal forms of Section 3, and formula (2) for the metric complexity still hold, as long as the fundamental 2-form does not vanish along $\Gamma$. This is Theorem 1.8. In fact, the results are the same, the only difference is that the metric complexity becomes non smooth: it has jumps on the second derivative. The strong asymptotic optimal synthesis turns out to be slightly more complicated.

In the Appendix (Sect. 7), we collect a few technical facts that are needed along the paper. Results similar to Lemma 7.1 can be found in the literature. But for the sake of completeness we provide a simple proof.

\section{Genericity Results}

We consider the set $S_{R}^{\infty}$ of relevant motion-planning problems $\Sigma=(F, \Gamma)$ with the $C^{\infty}$ topology, we consider also the field $\widetilde{\alpha}_{\Sigma}$ of fundamental 2 -forms along $\Gamma$ defined by $\Sigma$. As explained above, in the coordinates on $\Delta$ defined by the frame $\mathrm{F}$, to $\widetilde{\alpha}_{\Sigma}(\Gamma(t))$ corresponds a skew symmetric matrix $\bar{A}(t)$.

As customary, $\mathfrak{s o}(N-1)$ denotes the Lie algebra of square $(N-1) \times(N-1)$ skew symmetric matrices, we have the following result for relevant motion-planning problems.

Theorem 2.1. The mapping $\rho: S_{R}^{\infty} \times[0,1] \longrightarrow \mathfrak{s o}(N-1), \quad(\Sigma, t) \longmapsto \bar{A}(t)$ is a submersion.

Proof. Let us consider a fixed $\Sigma=(F, \Gamma) \in S_{R}^{\infty}$, and an arbitrary $t_{0} \in[0,1]$. It is always possible to choose a neighborhood $V_{t_{0}}$ of $\Gamma\left(t_{0}\right)$, and coordinates $(x, w)=\left(x_{1}, \ldots, x_{N-1}, w\right)$ on $V_{t_{0}}$ such that:

$$
\begin{aligned}
\Gamma(t) & =\left(0, t-t_{0}\right), \\
\Delta(0, w) & =\operatorname{ker} \mathrm{d} w .
\end{aligned}
$$

Furthermore, each vector field $F_{j}$ of the frame $F=\left(F_{1}, \ldots, F_{N-1}\right)$ can be written as $(4)$, with $L(0, w)=0$.

The matrix $Q(x, w)$ is invertible, because $\Gamma$ is transversal to $\Delta$ and $F$ is an orthonormal frame. Let $Q_{i j}^{-1}$ denote the $i j$ entry of the inverse matrix $\widetilde{\Omega}(x, w)=Q^{-1}(x, w)$.

Let $\Omega=\left(\omega_{1}, \ldots, \omega_{N}\right)$ be the coframe dual to the frame $\left(F_{1}, \ldots, F_{N-1}, \frac{\partial}{\partial w}\right)$. Then, by definition we have

$$
\left.\mathrm{d} \omega_{N}\right|_{\Delta(\Gamma(t))}=\widetilde{\alpha}(\Gamma(t))
$$

Set $L(x, w)=\left(L_{1}(x, w), \ldots, L_{N-1}(x, w)\right)$, the line vector with components $L_{1}, \ldots, L_{N-1}$. Then, if $\mathrm{d} x$ is the column vector with components $\mathrm{d} x_{1}, \ldots, \mathrm{d} x_{N-1}$, we have: 


$$
\begin{aligned}
\omega_{i} & =\widetilde{\Omega_{i}}(x, w) \mathrm{d} x, \quad i=1, \ldots, N-1, \\
\omega_{N} & =\widetilde{L}(x, w) \mathrm{d} x+\mathrm{d} w
\end{aligned}
$$

where $\widetilde{L}=-L Q^{-1}=-L \widetilde{\Omega}$, and $\widetilde{\Omega_{i}}$ is the $i$ th line of the matrix $\widetilde{\Omega}$.

Therefore

$$
\mathrm{d} \omega_{N}\left(F_{l}, F_{s}\right)=\sum_{i, j, k=1}^{N-1} \frac{\partial}{\partial x_{k}}\left(L_{i} Q_{i j}^{-1}\right)\left(Q_{j l} Q_{k s}-Q_{j s} Q_{k l}\right)+\sum_{i, j=1}^{N-1} \frac{\partial}{\partial w}\left(L_{i} Q_{i j}^{-1}\right)\left(L_{s} Q_{j l}-L_{l} Q_{j s}\right),
$$

and since $L(0, w)=0$, we have:

$$
\left.\mathrm{d} \omega_{N}\left(F_{l}, F_{s}\right)\right|_{\Gamma}=\sum_{i, j, k=1}^{N-1} \frac{\partial L_{i}}{\partial x_{k}} Q_{i j}^{-1}\left(Q_{j l} Q_{k s}-Q_{j s} Q_{k l}\right) .
$$

Let us fix $Q$, and leave $L$ free. By definition we know that

$$
\bar{A}_{s l}\left(t_{0}\right)=\mathrm{d} \omega_{N}\left(F_{l}, F_{s}\right)\left(\Gamma\left(t_{0}\right)\right)
$$

Let us consider the $(N-1) \times(N-1)$ matrices $\bar{L}$ and $\widehat{L}$, the entries of which are given as follows

$$
\begin{aligned}
\bar{L}_{j k} & =\sum_{i=1}^{N-1} \frac{\partial L_{i}}{\partial x_{k}} Q_{i j}^{-1}\left(\Gamma\left(t_{0}\right)\right), \\
\widehat{L}_{i k} & =\frac{\partial L_{i}}{\partial x_{k}}\left(\Gamma\left(t_{0}\right)\right) .
\end{aligned}
$$

Then $\bar{L}=\left(Q^{-1}\right)^{\prime} \widehat{L}\left(\Gamma\left(t_{0}\right)\right)$, and (8) can be rewritten:

$$
\bar{A}\left(t_{0}\right)=Q^{\prime} \bar{L}^{\prime} Q-Q^{\prime} \bar{L} Q=Q^{\prime}\left(\bar{L}^{\prime}-\bar{L}\right) Q
$$

where' denotes the corresponding transpose matrix. Then, $\bar{A}\left(t_{0}\right)=0$ implies $\bar{L}=\bar{L}^{\prime}$, which means that $\bar{L}$ is symmetric, and that $\widehat{L}=Q^{\prime}\left(\Gamma\left(t_{0}\right)\right) S$, where $S$ is any symmetric matrix.

Therefore, the kernel of the linear map

$$
\mathcal{M}_{N-1 \times N-1} \longrightarrow \mathfrak{s o}(N-1), \quad \widehat{L} \longmapsto \bar{A}\left(t_{0}\right),
$$

has dimension $\frac{1}{2}(N-1) N$. Hence, its image has dimension $\frac{1}{2}(N-1)(N-2)$ and consequently is surjective. It follows then that $\rho: S_{R}^{\infty} \times[0,1] \longrightarrow \mathfrak{s o}(N-1), \quad(\Sigma=(F, \Gamma), t) \longmapsto \bar{A}(t)$, is a submersion, as claimed.

We apply now standard transversality techniques (see for instance $[1,4,8]$ ), to the mapping $\rho$, and to the closed stratified subsets $\mathcal{A}_{1}, \mathcal{A}_{2}, \mathcal{A}_{3}, \overline{\mathcal{A}}_{4}$ of $\mathfrak{s o}(N-1)$ which are defined in the Appendix 7 , Section 7.2. There is an open and dense subset $S_{*}^{\infty} \subset S_{R}^{\infty}$ such that, for any $\Sigma \in S_{*}^{\infty}$ the mapping

$$
e v_{\rho}(\Sigma):[0,1] \longrightarrow \mathfrak{s o}(N-1), \quad t \longmapsto \rho(\Sigma, t),
$$

is transversal to the aforementioned closed stratified subsets of $\mathfrak{s o}(N-1)$. It is enough to evaluate the codimension of these sets to conclude that for $\Sigma \in S_{*}^{\infty}$ one has that

(1) for all $t \in[0,1], \quad \bar{A}(t)$ is not an element of $\overline{\mathcal{A}}_{4} \cup \mathcal{A}_{2} \cup \mathcal{A}_{3}$, and

(2) the set $\left\{t \in[0,1] \mid \bar{A}(t) \in \mathcal{A}_{1}\right\}$ is finite.

In conclusion we have the following result: 
Theorem 2.2. There is an open and dense subset $S_{*}^{\infty} \subset S_{R}^{\infty}$, such that for all $\Sigma \in S_{*}^{\infty}$, the mapping $t \mapsto A(t)$ has the following properties. For all $t \in[0,1]$ :

(1) $A(t)$ has no double nonzero eigenvalues;

(2) the kernel of $A(t)$ has dimension 1 if $N=2 n$;

(3) the kernel of $A(t)$ has dimension 2 on a (may be empty) finite subset of $[0,1]$, and it is zero elsewhere if $N=2 n+1$.

In particular, and this will be a key point in the next sections, for $\Sigma \in S_{*}^{\infty}$ and all $t \in[0,1]$, if $N \geq 4$ then $A(t)$ has a nonzero eigenvalue.

\section{NORMAL COORDINATES AND NORMAL FORMS}

The results in this section are very close to the results in the papers $[2,3,5,6]$. We refer the reader to these references, and limit ourselves to explain in detail the points that are different. We recall that a sub-Riemannian geodesic is by definition an admissible curve that minimizes the length functional given by (1). It is well known that Pontryagin maximum principle provides necessary conditions for sub-Riemannian geodesics.

\subsection{Normal coordinates}

Theorem 3.1. Let $\Sigma=(F, \Gamma) \in S_{R}^{\infty}$. Then there is a global coordinate system $(\xi, w) \in \mathbb{R}^{N-1} \times \mathbb{R}$, defined on a neighborhood of $\Gamma([0,1])$, such that:

(1) $\Gamma(t)=(0, t), \Delta(\Gamma(t))=$ ker $\mathrm{d} w$ and $\left.g\right|_{\Gamma(t)}=\sum_{i=1}^{N-1} \mathrm{~d} \xi_{i}^{2}$;

(2) geodesics satisfying the Pontryagin Maximum Principle's transversality conditions with respect to $\Gamma$ are the straight lines through $\Gamma$ contained in the planes $S_{w_{0}}=\left\{(\xi, w) \mid w=w_{0}\right\}$;

(3) for $\varepsilon$ small enough, the sub-Riemannian cylinder $C_{\varepsilon}=\{q \mid \mathrm{d}(q, \Gamma)=\varepsilon\}$ is the Riemannian cylinder $\left\{\|\xi\|_{2}=\varepsilon\right\}$, (here $d$ denotes the sub-Riemannian distance associated to $\Sigma$ ).

The coordinate system in the Theorem 3.1 is usually called a pre-normal coordinate system. The proof of this theorem is included in the aforementioned references as well as in [6]. The paper [5] discusses dimension 3, but in formal power series, whereas [2] does it in the smooth case. Reference [3] studies the contact case in any odd dimension whereas reference [6] treats the quasi-contact in any even dimension. In fact, there is no need for the contact or quasi-contact assumptions, since the only crucial point in those cases is that the curve $\Gamma$ has to be everywhere transversal to the distribution $\Delta$, which is, by definition, the case in $S_{R}^{\infty}$.

Now, in a pre-normal coordinate system $(\xi, w)$ along the curve $\Gamma$, the vector fields

$$
\frac{\partial}{\partial \xi_{1}}, \ldots, \frac{\partial}{\partial \xi_{N-1}}
$$

provide an orthonormal frame of $\Delta(\Gamma(t))$. Let $\bar{A}(t)$ be the matrix of the linear map $A(t)$ with respect to this frame.

Theorem 3.2 (normal coordinates). Let $\Sigma=(F, \Gamma) \in S_{*}^{\infty}$. Then, there is a global coordinate system $(\xi, w)$, defined on a neighborhood of $\Gamma([0,1])$, such that:

(1) $(\xi, w)$ is a pre-normal coordinate system;

(2) in the coordinates $\xi$, the matrices $\bar{A}(t), t \in[0,1]$ are (skew-symmetric) $2 \times 2$ block-diagonal. (Plus a $1 \times 1$ block if $N=2 n$.)

Proof. By Lemma 7.1, Appendix 7, we can smoothly block-diagonalize the matrix $\bar{A}(t)$ along the curve $\Gamma$, using a smooth orthogonal transformation $U(t)$. Now, setting $(\widetilde{\xi}, w)=(U(w) \xi, w)$ we obtain the desired coordinates, because such a change of coordinates does not affect the properties of pre-normal coordinates in Theorem 3.1. 
Normal coordinates are unique up to a change of coordinates of the form:

$$
(\widetilde{\xi}, w)=(T(w) \xi, w)
$$

where $T(w)$ is a smooth curve in the natural maximal-torus of the orthogonal group $\operatorname{SO}(N-1)$.

In the contact and quasi-contact cases, we can do even more, because in those cases we can consider, in normal coordinates, the planes $S_{w_{0}}=\left\{(\xi, w) \mid w=w_{0}\right\}$. Since the curve $\Gamma$ is transversal to $\Delta$, then the subRiemannian metric $g(\xi, w)$ projects on a Riemannian metric $g_{w}$ on the plane $S_{w}$. In fact, if $\Pi_{w_{0}}$ denotes the projection

$$
\Pi_{w_{0}}: \Xi_{N} \rightarrow S_{w_{0}}, \quad(\xi, w) \mapsto\left(\xi, w_{0}\right),
$$

we set

$$
g_{w_{0}}(X, Y)=g\left(\left(\left.\mathrm{~d} \Pi_{w_{0}}\right|_{\Delta}\right)^{-1} X,\left(\left.\mathrm{~d} \Pi_{w_{0}}\right|_{\Delta}\right)^{-1} Y\right), \quad \text { for } \quad X, Y \in T_{\left(\xi, w_{0}\right)} S_{w_{0}} .
$$

Theorem 3.3. If $\Delta$ is contact or quasi-contact, we can chose $T(w)$ in such a way that for all points (0, $w)$ on the curve $\Gamma$, the sectional curvatures of the metrics $g_{w}$, with respect to the 2-dimensional real eigenspaces of $A(w)$ are zero.

This theorem is proved in [2] (3-dimensional case), [3] (general contact case), and [6] (quasi-contact case). These normal coordinates are again unique, up to an element $T\left(w_{0}\right)$ of a maximal torus of $\mathrm{SO}(N-1)$. (Once the $T\left(w_{0}\right)$ in $(11)$ is fixed for some $w_{0}$, then, it determines $T(w)$ for all $w$, for the sectional curvatures being zero.)

\subsection{Normal forms}

Assume that $\Sigma=(F, \Gamma)$ is given, we write the normal coordinates $(\xi, w)$ along the curve $\Gamma$ according to the following notation: $(\xi, w)=(x, y, w)$, for $N=2 n+1$; and $(\xi, w)=(x, y, z, w)$, for $N=2 n$. Clearly $\xi=(x, y)$ and $\xi=(x, y, z)$ respectively, and

$$
\begin{array}{ll}
(x, y)=\left(x_{1}, y_{1}, \ldots, x_{n}, y_{n}\right), & \text { for } N=2 n+1, \text { whereas } \\
(x, y)=\left(x_{1}, y_{1}, \ldots, x_{n-1}, y_{n-1}\right), & \text { for } N=2 n
\end{array}
$$

Then, as in Section 2, formula (4), we write in coordinates $\Sigma=(Q(\xi, w), L(\xi, w), \Gamma)$.

We expand $Q(\xi, w)$ and $L(\xi, w)$ in power series of the variables $\xi_{j}$, at $\xi=0$, along $\Gamma$, as follows:

$$
\begin{aligned}
& Q(\xi, w)=Q_{0}(\xi, w)+Q_{1}(\xi, w)+Q_{2}(\xi, w)+\cdots \\
& L(\xi, w)=L_{0}(\xi, w)+L_{1}(\xi, w)+L_{2}(\xi, w)+\cdots
\end{aligned}
$$

where $Q_{k}$ and $L_{k}$ are homogeneous polynomial matrices of degree $k$, in the variables $x_{j}$ and $y_{j}$ (and $z$, if $N=2 n)$. Then, for $\Sigma=(F, \Gamma) \in S_{*}^{\infty}$, setting $i=\sqrt{-1}$, we know the following two facts:

a) for $N=2 n$, the eigenvalues of $A(w)$ are smooth functions: $\pm i \alpha_{1}(w), \ldots, \pm i \alpha_{n-1}(w), 0$, where $\alpha_{j}(w)>0$ for all $j$. For all $w$, we have that $\alpha_{k}(w) \neq \alpha_{l}(w)$, provided $k \neq l$. Hence, for all $w$, we can order the eigenvalues as follows:

$$
\alpha_{1}(w)>\cdots>\alpha_{n-1}(w)>0
$$

b) for $N=2 n+1$, the eigenvalues of $A(w)$ are smooth functions: $\pm i \alpha_{1}(w), \ldots, \pm i \alpha_{n}(w)$, where again for all $w$ we have that $\alpha_{k}(w) \neq \pm \alpha_{l}(w)$ for $k \neq l$, and one at most is zero, for isolated values of $w$. If none of the $\alpha_{j}^{\prime} s$ vanishes somewhere, we proceed as in a). If one vanishes for some $w_{0}$, then, we order the eigenvalues at $w_{0}$, as follows:

$$
\alpha_{1}\left(w_{0}\right)>\cdots>\alpha_{n-1}\left(w_{0}\right)>\alpha_{n}\left(w_{0}\right)=0
$$


in any case, we can always conclude that for all $w$ we have

$$
\alpha_{1}(w)>\cdots>\alpha_{n-1}(w)>\alpha_{n}(w)
$$

and $\alpha_{n-1}(w)>0$ for all $w$. This last point $\left(\alpha_{n-1}(w)>0\right)$ holds, because if it were not true, at some point $w^{\prime}$, then $\alpha_{n-1}\left(w^{\prime}\right)= \pm \alpha_{n}\left(w^{\prime}\right) \neq 0$, which is not possible.

In the normal coordinates, it might be eventually necessary to consider permutations $\left(x_{j}, y_{j}\right) \rightarrow\left(y_{j}, x_{j}\right)$, to write:

$$
\begin{aligned}
& \left.\mathrm{d} \omega\right|_{\Delta(\Gamma(t))}=\alpha_{1}(t) \mathrm{d} x_{1} \wedge \mathrm{d} y_{1}+\cdots+\alpha_{n}(t) \mathrm{d} x_{n} \wedge \mathrm{d} y_{n}, \quad \text { and } \\
& \left.\mathrm{d} \omega\right|_{\Delta(\Gamma(t))}=\alpha_{1}(t) \mathrm{d} x_{1} \wedge \mathrm{d} y_{1}+\ldots+\alpha_{n-1}(t) \mathrm{d} x_{n-1} \wedge \mathrm{d} y_{n-1}
\end{aligned}
$$

for $N=2 n+1$, and $N=2 n$ respectively.

Here the frame (4) along the curve $\Gamma$ is orthonormal, as a frame of $\Delta$, and the matrices $\bar{A}(t)$ of the linear map $A(t)$, with respect to this frame are the following:

$$
\bar{A}(w)=\left(\begin{array}{ccccccc}
0 & -\alpha_{1}(w) & 0 & 0 & \cdots & 0 & 0 \\
\alpha_{1}(w) & 0 & 0 & 0 & \cdots & 0 & 0 \\
0 & 0 & 0 & -\alpha_{2}(w) & \cdots & 0 & 0 \\
0 & 0 & \alpha_{2}(w) & 0 & \cdots & 0 & 0 \\
\vdots & \vdots & \vdots & \vdots & \ddots & \vdots & \vdots \\
0 & 0 & 0 & 0 & \cdots & 0 & -\alpha_{n}(w) \\
0 & 0 & 0 & 0 & \cdots & \alpha_{n}(w) & 0
\end{array}\right)
$$

for $N=2 n+1$, and

$$
\bar{A}(w)=\left(\begin{array}{cccccccc}
0 & -\alpha_{1}(w) & 0 & 0 & \cdots & 0 & 0 & 0 \\
\alpha_{1}(w) & 0 & 0 & 0 & \cdots & 0 & 0 & 0 \\
0 & 0 & 0 & -\alpha_{2}(w) & \cdots & 0 & 0 & 0 \\
0 & 0 & \alpha_{2}(w) & 0 & \cdots & 0 & 0 & 0 \\
\vdots & \vdots & \vdots & \vdots & \ddots & \vdots & \vdots & \vdots \\
0 & 0 & 0 & 0 & \cdots & 0 & -\alpha_{n-1}(w) & 0 \\
0 & 0 & 0 & 0 & \cdots & \alpha_{n-1}(w) & 0 & 0 \\
0 & 0 & 0 & 0 & \cdots & 0 & 0 & 0
\end{array}\right)
$$

for $N=2 n$.

Theorem 3.4 (normal form). Let $\Sigma=(F, \Gamma) \in S_{*}^{\infty}$, and let $(\xi, w)=(x, y, w)$ or $(\xi, w)=(x, y, z, w)$, be a fixed normal coordinate system. Then, there is a unique sub-Riemannian orthonormal frame $\mathcal{F}=(Q, L)$, (gauge equivalent to $F$ ), with the following properties:
1) $Q$ is symmetric;
2) $Q_{0}(\xi, w)=I d$;
3) $Q(\xi, w) \xi=\xi$;
4) $L(\xi, w) \cdot \xi=0$;
5) $Q_{1}=0$;
6) $L_{0}=0$; 
7) the first order linear forms are given as follows:

$$
\begin{aligned}
& L_{1}=\left(\frac{\alpha_{1}(w)}{2} y_{1},-\frac{\alpha_{1}(w)}{2} x_{1}, \ldots, \frac{\alpha_{n}(w)}{2} y_{n},-\frac{\alpha_{n}(w)}{2} x_{n}\right), \text { and } \\
& L_{1}=\left(-\frac{\alpha_{1}(w)}{2} y_{1},-\frac{\alpha_{1}(w)}{2} x_{1}, \ldots, \frac{\alpha_{n-1}(w)}{2} y_{n-1}, \frac{\alpha_{n-1}(w)}{2} x_{n-1}, 0\right),
\end{aligned}
$$

for $N=2 n+1$ and $N=2 n$ respectively.

Proof. The identities 1) to 6) are proved in [3] for the contact case and in [6] for the quasi-contact one. Equation 5) is an interesting sub-Riemannian analog of a Bianchi identity in Riemannian geometry. We prove 7 ), which is the only different point. We prove it for $N=2 n+1$ only, (the case $N=2 n$ is similar).

Here, $\mathrm{d} \xi$ is the column vector with components $\mathrm{d} \xi_{1}, \ldots, \mathrm{d} \xi_{N-1}$. We already know (Sect. 2, proof of Th. 2.1) that, if we take $\omega_{N}=\mathrm{d} w-L Q^{-1} \mathrm{~d} \xi$, then $\widetilde{\alpha}(\Gamma(w))=\left.\mathrm{d} \omega_{N}\right|_{\Delta(\Gamma(w))}$ has $\bar{A}(w)$ as matrix. Now, on one hand, by definition of our coordinates, we have that

$$
\left.\mathrm{d} \omega_{N}\right|_{\Delta(\Gamma(w))}=\alpha_{1}(w) \mathrm{d} x_{1} \wedge \mathrm{d} y_{1}+\cdots+\alpha_{n}(w) \mathrm{d} x_{n} \wedge \mathrm{d} y_{n}
$$

and on the other hand that

$$
\left.\mathrm{d} \omega_{N}\right|_{\Delta(\Gamma(w))}=-\mathrm{d}\left(L Q^{-1} \mathrm{~d} \xi\right)(\Gamma(w)) .
$$

Now, (6) implies

$$
L=L_{1}(\xi, w)+O^{2}(\xi)=\left(L_{1}^{1}, M_{1}^{1}, \ldots, L_{1}^{n}, M_{1}^{n}\right)+O^{2}(\xi),
$$

and in consequence 4) yields,

$$
L_{1}^{1} x_{1}+M_{1}^{1} y_{1}+\cdots+L_{1}^{n} x_{n}+M_{1}^{n} y_{n}=0
$$

Therefore, by writing for all $k$ :

$$
\begin{aligned}
L_{1}^{k} & =\sum_{j}\left(L_{1}^{k j} x_{j}+\widehat{L_{1}^{k j}} y_{j}\right), \quad \text { and } \\
M_{1}^{k} & =\sum_{j}\left(M_{1}^{k j} x_{j}+\widehat{M_{1}^{k j}} y_{j}\right),
\end{aligned}
$$

we obtain

$$
\sum_{j, k}\left(L_{1}^{k j} x_{j} x_{k}+\widehat{L_{1}^{k j}} y_{j} x_{k}+M_{1}^{k j} x_{j} y_{k}+\widehat{M_{1}^{k j}} y_{j} y_{k}\right)=0
$$

which implies:

$$
L_{1}^{k k}=0, \quad \widehat{M_{1}^{k k}}=0, \quad L_{1}^{k j}=-L_{1}^{j k}, \quad \widehat{L_{1}^{k j}}=-M_{1}^{j k}, \quad \text { and } \quad \widehat{M_{1}^{k j}}=-\widehat{M_{1}^{j k}} .
$$

A straightforward calculation yields

$$
\left.\mathrm{d}\left(L_{1} \mathrm{~d} \xi\right)\right|_{\Gamma}=2 \sum_{j<k} L_{1}^{k j} \mathrm{~d} x_{j} \wedge \mathrm{d} x_{k}+2 \sum_{j<k} \widehat{M_{1}^{k j}} \mathrm{~d} y_{j} \wedge \mathrm{d} y_{k}+\sum_{j, k} \widehat{L_{1}^{k j}} \mathrm{~d} y_{j} \wedge \mathrm{d} x_{k}+\sum_{j, k} M_{1}^{k j} \mathrm{~d} x_{j} \wedge \mathrm{d} y_{k},
$$

or equivalently

$$
\left.\mathrm{d}\left(L_{1} \mathrm{~d} \xi\right)\right|_{\Gamma}=2\left(\sum_{j<k}\left(L_{1}^{k j} \mathrm{~d} x_{j} \wedge \mathrm{d} x_{k}+\widehat{M_{1}^{k j}} \mathrm{~d} y_{j} \wedge \mathrm{d} y_{k}\right)-\sum_{j, k} \widehat{L_{1}^{k j}} \mathrm{~d} x_{k} \wedge \mathrm{d} y_{j}\right)
$$


Now,

$$
-\left.\mathrm{d}\left(L Q^{-1} \mathrm{~d} \xi\right)\right|_{\Delta(\Gamma(w))}=-\left.\mathrm{d}(L \mathrm{~d} \xi)\right|_{\Delta(\Gamma(w))},
$$

(because $Q^{-1}=I d+O^{2}(\xi)$ ), and,

$$
-\left.\mathrm{d}(L \mathrm{~d} \xi)\right|_{\Delta(\Gamma(w))}=-\left.\mathrm{d}\left(L_{1} \mathrm{~d} \xi\right)\right|_{\Delta(\Gamma(w))} .
$$

By (14), we deduce that for all $k$ and $j$

$$
\begin{aligned}
\frac{L_{1}^{k j}}{M_{1}^{k j}} & =0, \\
2 \sum_{j, k} \widehat{L_{1}^{k j} \mathrm{~d} x_{k} \wedge \mathrm{d} y_{j}} & =-\sum_{j} \alpha_{j}(w) \mathrm{d} x_{j} \wedge \mathrm{d} y_{j} .
\end{aligned}
$$

Hence,

$$
\begin{aligned}
& \widehat{L_{1}^{k j}}=0, \quad \text { for } \quad k \neq j, \quad \text { and } \\
& \widehat{L_{1}^{k k}}=-M_{1}^{k k}=-\frac{\alpha_{k}}{2} .
\end{aligned}
$$

This gives (7), changing $\left(x_{i}, y_{i}\right)$ for $\left(y_{i}, x_{i}\right)$.

Conversely, if we have a coordinate neighborhood of $\Gamma$ and a frame $F$ meeting conditions 1), 2), 3), 4), 7), then 5),6) are automatically satisfied, and the coordinates turn out to be normal.

\subsection{Special case: normal form in dimension 3}

In dimension 3 , the normal form is given by the frame $F=\left\{F_{1}, G_{1}\right\}$

$$
\begin{aligned}
F_{1} & =\left(1+y^{2} \beta(x, y, w)\right) \frac{\partial}{\partial x}-x y \beta(x, y, w) \frac{\partial}{\partial y}+\frac{y}{2} \gamma(x, y, w) \frac{\partial}{\partial w} \\
G_{1} & =-x y \beta(x, y, w) \frac{\partial}{\partial x}+\left(1+x^{2} \beta(x, y, w)\right) \frac{\partial}{\partial y}-\frac{x}{2} \gamma(x, y, w) \frac{\partial}{\partial w}
\end{aligned}
$$

where $\beta$ and $\gamma$ are smooth functions.

In the contact case, $\gamma(0,0, w)$ never vanishes, and we can take it positive. Furthermore, we can normalize the function $\beta$ by the condition $\beta(0,0, w)=0$, which is tantamount of saying that the curvature or the metric $g_{w}$, (projection of $g$ on the cross section $S_{w}=\{w=$ constant $\}$ ), is zero. This normal form has been first given in [5], for the formal power series case, and after in [2] for the smooth case.

\section{Complexity AND ASYMPtotic Optimal Synthesis}

We discuss now the metric complexity. The results of this section are valid for all $N \geq 4$, and in the absence of Martinet points along the curve $\Gamma$, they are also valid for $N=3$.

Using Theorem 3.4 (normal form), and the notations of Section 3, we consider a normal coordinate neighborhood $(\xi, w)$ of the curve $\Gamma$, and a normal frame along $\Gamma$, that we write as:

$$
\begin{aligned}
& F=\left(F_{1}, G_{1}, \ldots, F_{n}, G_{n}\right), \quad \text { for } \quad N=2 n+1 \text { or } \\
& F=\left(F_{1}, G_{1}, \ldots, F_{n-1}, G_{n-1}, H_{n}\right), \text { for } N=2 n
\end{aligned}
$$


where

$$
\begin{aligned}
F_{i} & =\left(1+O^{2}(\xi)\right) \frac{\partial}{\partial x_{i}}+\left(\frac{y_{i}}{2} \alpha_{i}(w)+O^{2}(\xi)\right) \frac{\partial}{\partial w}+O^{2}(\xi) \\
G_{i} & =\left(1+O^{2}(\xi)\right) \frac{\partial}{\partial y_{i}}+\left(-\frac{x_{i}}{2} \alpha_{i}(w)+O^{2}(\xi)\right) \frac{\partial}{\partial w}+O^{2}(\xi), \\
H_{n} & =\left(1+O^{2}(\xi)\right) \frac{\partial}{\partial x_{N-1}}+O^{2}(\xi) \frac{\partial}{\partial w}+O^{2}(\xi) .
\end{aligned}
$$

Here, $O^{i}(\xi)$ are different smooth functions or vector fields in the variables $(\xi, w)$, that are in the $i$ th power of the ideal generated by $\xi_{i}, i=1, \ldots, N-1$.

Let us now consider any (smooth) admissible curve $\delta:[0, T] \rightarrow T_{\varepsilon}, t \mapsto \delta(t)=(\widehat{\xi}(t), \widehat{w}(t))$ entirely contained in the sub-Riemannian tube $T_{\varepsilon}$, (hence, in normal coordinates, contained in the Riemannian tube, because in normal coordinates, they are the same). Furthermore, let us assume that the following conditions hold:

$$
\widehat{w}(0)=w_{0}, \quad \text { and } \quad \widehat{w}(T)=w_{1}
$$

Assume that the curve $\delta$ is parameterized by arc length, assume also that the corresponding control functions are $u_{i}(\cdot)$ with $i=1, \ldots, N-1$. The length of $\delta$ is $T$, and

$$
\sum_{i=1}^{N-1} u_{i}^{2}(t)=1, \quad \text { for all } t \in[0, T]
$$

and also

$$
\frac{\mathrm{d} \widehat{w}}{\mathrm{~d} t}=\sum_{i=1}^{N-1}\left(L_{i}^{1}(\widehat{\xi}, \hat{w})+O^{2}(\hat{\xi})\right) u_{i} .
$$

Let $\kappa(w)=\sup _{i}\left\{\left|\alpha_{i}(w)\right|\right\}$, we have that $\kappa$ is strictly positive on the interval $\left[w_{0}, w_{1}\right]$. Furthermore, for $N=2 n+1$ we have that

$$
\frac{\mathrm{d} \widehat{w}}{\mathrm{~d} t} \leq \frac{\kappa(\widehat{w})}{2}\left(\left|\widehat{y}_{1}\right|\left|u_{1}\right|+\left|\widehat{x}_{1}\right|\left|u_{2}\right|+\cdots+\left|\widehat{y}_{n}\right|\left|u_{N-2}\right|+\left|\widehat{x}_{n}\right|\left|u_{N-1}\right|\right)+\left|O^{2}(\widehat{\xi})\right|,
$$

whereas for $N=2 n$

$$
\begin{aligned}
\frac{\mathrm{d} \widehat{w}}{\mathrm{~d} t} & \leq \frac{\kappa(\widehat{w})}{2}\left(\left|\widehat{y}_{1}\right|\left|u_{1}\right|+\left|\widehat{x}_{1}\right|\left|u_{2}\right|+\cdots+\left|\widehat{y}_{n-1}\right|\left|u_{N-3}\right|+\left|\widehat{x}_{n-1}\right|\left|u_{N-2}\right|\right)+\left|O^{2}(\widehat{\xi})\right| \\
& \leq \frac{\kappa(\widehat{w})}{2}\langle\widehat{\xi}|,| u \mid\rangle+O^{2}(\widehat{\xi})
\end{aligned}
$$

where $|\widehat{\xi}|$ and $|u|$ are the vectors with entries $\left|\widehat{\xi}_{i}\right|$ and $\left|u_{i}\right|$ respectively. In any case, since $\widehat{\xi}$ is in the tube $T_{\varepsilon}$, and $\|u\|_{2}=1$, we have that

$$
\frac{\mathrm{d} \widehat{w}}{\mathrm{~d} t} \leq \frac{\kappa(\widehat{w})}{2}\left\|\widehat{\xi}\left|\left\|_{2}\right\|\right| u\left|\|_{2}+\right| O^{2}(\widehat{\xi}) \mid \leq \varepsilon \frac{\kappa(\widehat{w})}{2}+K \varepsilon^{2}\right.
$$

for some constant $K>0$. But then, there is a constant $K^{\prime}$ such that

$$
\frac{\mathrm{d} \widehat{w}}{\kappa(\widehat{w})} \leq \varepsilon \frac{\mathrm{d} t}{2}\left(1+\varepsilon K^{\prime}\right)
$$


and consequently, there is a constant $K^{\prime \prime}$, such that

$$
T \geq \frac{2}{\varepsilon} \int_{w_{0}}^{w_{1}} \frac{\mathrm{d} w}{\kappa(w)}\left(1-\varepsilon K^{\prime \prime}\right)
$$

Remark 4.1. In order to reduce the burden in the notation for the constants appearing in the estimates $\left(K, K^{\prime}, \ldots\right)$, in the remaining of the paper, we shall abuse the notation using only one $K$ for all the (different) constants.

Now, we shall exhibit a (strong) asymptotic optimal synthesis. We know that, for $\varepsilon$ small enough, the cylinder $C_{\varepsilon}=\{\|\xi\|=\varepsilon\}$ is transversal to the curve $\Gamma$. Let us consider also the following 2-dimensional cylinder

$$
C_{\varepsilon}^{1}=\left\{\xi \in C_{\varepsilon} \mid x_{1}^{2}+y_{1}^{2}=\varepsilon^{2}\right\} .
$$

Then, for $\varepsilon$ small enough, we have a (never vanishing) vector field $X_{\varepsilon}$ on $C_{\varepsilon}^{1}$ defined by the following three conditions:

a) $X_{\varepsilon}(\xi, w) \in \Delta(\xi, w) \cap T_{(\xi, w)}\left(C_{\varepsilon}^{1}\right)$;

b) $\left\|X_{\varepsilon}(\xi, w)\right\|_{g}=1$; and

c) $L_{X_{\varepsilon}}(w)>0$.

On the cylinder $C_{\varepsilon}^{1}$, the integral curves of $X_{\varepsilon}$ satisfy:

$$
\dot{w}=\frac{\alpha_{1}(w)}{2} \varepsilon+\varepsilon^{2} F(\varepsilon, \theta, w)
$$

where $F$ is a bounded function; and $x_{1}=\varepsilon \cos \theta, y_{1}=\varepsilon \sin \theta$. This can be shown by elementary but tedious calculations using the normal form. This shows that, for $\varepsilon$ sufficiently small, trajectories of $X_{\varepsilon}$ can join any $w_{0}$ and $w_{1}$ with $w_{0}<w_{1}$ and $\left(0, w_{0}\right),\left(0, w_{1}\right) \in T_{\varepsilon}$.

But then, since $F$ is bounded on $T_{\varepsilon}$ and $\left|\alpha_{1}(w)\right|>A>0$ for some constant $A$, there is a positive constant $K$ such that

$$
\frac{\dot{w}}{\alpha_{1}(w)}=\varepsilon\left(\frac{1}{2}+\varepsilon \frac{F(\varepsilon, \theta, w)}{\alpha_{1}}\right) \geq \frac{\varepsilon}{2}-\frac{\varepsilon^{2} K}{2} .
$$

In consequence, if $T(\varepsilon)$ is the time to join $w_{0}$ to $w_{1}$, then:

$$
\int_{w_{0}}^{w_{1}} \frac{\mathrm{d} w}{\alpha_{1}(w)} \geq \frac{\varepsilon}{2}(1-\varepsilon K) T(\varepsilon)
$$

and furthermore

$$
T(\varepsilon) \leq \frac{2}{\varepsilon} \frac{1}{(1-\varepsilon K)} \int_{w_{0}}^{w_{1}} \frac{\mathrm{d} w}{\kappa(w)} \leq \frac{2}{\varepsilon} \int_{w_{0}}^{w_{1}} \frac{\mathrm{d} w}{\kappa(w)}(1+\varepsilon A),
$$

for some $A>0$, if $\varepsilon$ is small enough.

Now, we can apply this to $w_{0}$ and $w_{1}$, such that:

$$
\Gamma(0)=\left(0, w_{0}\right), \quad \Gamma(1)=\left(0, w_{1}\right) .
$$

Our trajectory does not join $\Gamma(0)$ to $\Gamma(1)$, since it is entirely contained in $C_{\varepsilon}$. But then, any $(\xi, w) \in C_{\varepsilon}$ can be joined to $(0, w)$ in time $\varepsilon$, by definition of $C_{\varepsilon}$, and by the fact that the rays through $\Gamma$ in the planes $S_{w}$ are geodesics. Therefore, we can construct an admissible trajectory $\widehat{\delta}$ from $\Gamma(0)$ to $\Gamma(1)$, entirely contained in $T_{\varepsilon}$, such that the time $T(\varepsilon)$ to join $\Gamma(0)$ to $\Gamma(1)$ verifies:

$$
T(\varepsilon) \leq \frac{2(1+\varepsilon A)}{\varepsilon} \int_{w_{0}}^{w_{1}} \frac{\mathrm{d} w}{\kappa(w)}+2 \varepsilon .
$$


Since both curves are parameterized by arc-length, then (18) together with (17), imply

$$
\frac{2(1-\varepsilon K)}{\varepsilon^{2}} \int_{w_{0}}^{w_{1}} \frac{\mathrm{d} w}{\kappa(w)} \leq M C_{\Sigma}(\varepsilon) \leq \frac{2(1+\varepsilon A)}{\varepsilon^{2}} \int_{w_{0}}^{w_{1}} \frac{\mathrm{d} w}{\kappa(w)}+2 .
$$

As a conclusion we have that

$$
M C_{\Sigma}(\varepsilon) \stackrel{s}{\simeq} \frac{2}{\varepsilon^{2}} \int_{w_{0}}^{w_{1}} \frac{\mathrm{d} w}{\kappa(w)}
$$

which finishes the proof of Theorem 1.6 and also that of Theorem 1.7 in the absence of Martinet points. Even more, we have explicitly exhibited an asymptotic optimal synthesis that goes under the following steps:

a) follow a horizontal geodesic, to go from $\Gamma(0)$ to $C_{\varepsilon}^{1}$;

b) follow then the flow of $X_{\varepsilon}$ on $C_{\varepsilon}^{1}$ up to $w_{1}$;

c) connect the point reached on $C_{\varepsilon}^{1}$ to $\Gamma(1)$ by a horizontal geodesic.

\section{Martinet CASE}

\subsection{Proof of the Theorem $\mathbf{1 . 7}$}

Now, we consider in $\mathbb{R}^{3}$ the orthonormal frame $\left\{F_{1}, G_{1}\right\}$, in normal form (15),

$$
\begin{aligned}
F_{1} & =\left(1+y^{2} \beta(x, y, w)\right) \frac{\partial}{\partial x}-x y \beta(x, y, w) \frac{\partial}{\partial y}+\frac{y}{2} \gamma(x, y, w) \frac{\partial}{\partial w} \\
G_{1} & =-x y \beta(x, y, w) \frac{\partial}{\partial x}+\left(1+x^{2} \beta(x, y, w)\right) \frac{\partial}{\partial y}-\frac{x}{2} \gamma(x, y, w) \frac{\partial}{\partial w}
\end{aligned}
$$

Let us assume the existence of a single isolated Martinet point, at $q=(x, y, w)=0$ (we know, generically that Martinet points are isolated). Then:

$$
\gamma(x, y, w)=\gamma_{0}(w)+(x, y) \cdot \gamma_{1}(x, y, w), \quad \text { and } \quad \gamma_{0}(0)=0
$$

here and from now $(x, y) \cdot \gamma_{1}$ means the scalar product $x \gamma_{1}^{1}+y \gamma_{1}^{2}$.

For a generic problem $\Sigma=\left(F_{1}, G_{1}, \Gamma\right)$, it is easy to check (we leave this to the reader), that $\frac{\partial \gamma_{0}}{\partial w}$ is non zero at the Martinet point. In fact, $\left|\frac{\partial \gamma_{0}}{\partial w}(0)\right|$ is an invariant of the problem.

We can change the parameterization of $\Gamma$ using a smooth diffeomorphism $\Phi$ in such a way that, setting $\widetilde{w}=\Phi(w)$, we get:

$$
\gamma_{0}(\widetilde{w}) \frac{\partial}{\partial \widetilde{w}}=-\kappa \widetilde{w} \frac{\partial}{\partial \widetilde{w}}
$$

In what follows, we shall forget about $\widetilde{w}$, and we shall take,

$$
\gamma_{0}(w)=-\kappa w
$$

Let us assume $\kappa>0$ (the case $\kappa<0$ is similar). Since the admissible curves are parameterized by arc length, the controls $u(t)$ and $v(t)$ satisfy $u^{2}(t)+v^{2}(t)=1$, and for a trajectory entirely contained in the tube $T_{\varepsilon}$, we have:

$$
\frac{\mathrm{d} w}{\mathrm{~d} t}=\left(-\kappa w+(x, y) \cdot \gamma_{1}(x, y, w)\right)\left(\frac{y}{2} u-\frac{x}{2} v\right)
$$

Let us take an admissible curve $q(t)=(x(t), y(t), w(t))$, such that $w_{0}=w(0)<0<w(T)=w_{1}$. And let us consider $t_{1}$ and $t_{2}$ such that $0<t_{1} \leq t_{2}<T, w\left(t_{1}\right)$ is zero for the first time, and $w\left(t_{2}\right)$ is zero for the last time (continuity of $q(t))$. 
Let us consider the piece $w(\cdot)>0$ of the curve (the piece $w(\cdot)<0$ is similar) and assume that $q(t)$ is contained in the $\varepsilon$-tube $T_{\varepsilon}$. Then, on $\left[t_{2}, T\right]$, we have:

$$
\left|\frac{y}{2} u-\frac{x}{2} v\right| \leq \frac{\varepsilon}{2}, \quad \text { and } \quad \frac{\mathrm{d} w}{\mathrm{~d} t} \leq(\kappa w+\varepsilon K) \frac{\varepsilon}{2},
$$

since $\gamma_{1}(x, y, w)$ is bounded on the compact tube $T_{\varepsilon}$.

Now, since $w(\cdot)$ is at least absolutely continuous then,

$$
\widetilde{w}(t)=w(t) \mathrm{e}^{-\frac{\kappa}{2} \varepsilon\left(t-t_{2}\right)}
$$

is also absolutely continuous. A direct estimation yields

$$
\frac{\mathrm{d} \widetilde{w}}{\mathrm{~d} t}=\frac{\mathrm{d} w}{\mathrm{~d} t} \mathrm{e}^{-\frac{\kappa}{2} \varepsilon\left(t-t_{2}\right)}-\frac{\kappa \varepsilon}{2} \widetilde{w} \leq \frac{\kappa \varepsilon}{2} \widetilde{w}+\frac{\varepsilon^{2}}{2} K \mathrm{e}^{-\frac{\kappa}{2}\left(t-t_{2}\right)}-\frac{\kappa \varepsilon}{2} \widetilde{w},
$$

but then

$$
\frac{\mathrm{d} \widetilde{w}}{\mathrm{~d} t} \leq \frac{\varepsilon^{2}}{2} K \mathrm{e}^{-\frac{\kappa}{2} \varepsilon\left(t-t_{2}\right)}, \quad \text { and } \quad \widetilde{w}(t) \leq \frac{\varepsilon}{\kappa} K\left(1-\mathrm{e}^{-\frac{\kappa}{2} \varepsilon\left(t-t_{2}\right)}\right)
$$

therefore

which means that:

$$
w(t) \leq \frac{\varepsilon}{\kappa} K\left(\mathrm{e}^{\frac{\kappa \varepsilon}{2}\left(t-t_{2}\right)}-1\right) \leq \frac{\varepsilon}{\kappa} K \mathrm{e}^{\frac{\kappa \varepsilon}{2}\left(t-t_{2}\right)},
$$

$$
\left(t-t_{2}\right) \geq \frac{2}{\varepsilon \kappa} \ln \left(\frac{\kappa w(t)}{\varepsilon K}\right)=-\frac{2}{\kappa}\left(\frac{\ln \varepsilon}{\varepsilon}-\frac{\ln \left(\frac{\kappa w(t)}{K}\right)}{\varepsilon}\right) .
$$

For the part of the curve corresponding to $w(t)<0$, we get a similar estimation (changing $w$ for $-w$ in $(20$ ) above, the computations are similar). At the end, if $T$ is the time to connect $w_{0}$ to $w_{1}$, we obtain that:

$$
T \geq-\frac{4}{\kappa} \frac{\ln \varepsilon}{\varepsilon}+\frac{2}{\varepsilon \kappa} \ln \left(\frac{\kappa^{2} w_{0} w_{1}}{K^{2}}\right),
$$

which shows that:

$$
M C_{\Sigma}(\varepsilon) \geq-\frac{4}{\kappa} \frac{\ln \varepsilon}{\varepsilon^{2}}+\frac{2}{\varepsilon^{2} \kappa} \ln \left(\frac{\kappa^{2} w_{0} w_{1}}{K^{2}}\right) .
$$

Now if we take any constant $C$ such that $0<C<\frac{4}{\kappa}$, for $\varepsilon$ small enough then

$$
M C_{\Sigma}(\varepsilon) \geq-C \frac{\ln \varepsilon}{\varepsilon^{2}}
$$

And the proof of Theorem 1.7 is complete.

Remark 5.1. In fact, it can be proved that in general,

$$
M C_{\Sigma}(\varepsilon) \stackrel{s}{\simeq}-C \frac{\ln \varepsilon}{\varepsilon^{2}}, \quad \text { where } \quad \frac{4}{\kappa} \leq C \leq \frac{12}{\kappa}
$$

however this estimation requires tedious calculations, that shall be discussed elsewhere. We limit ourselves to analyze an example, for which the estimation (22) above holds. 


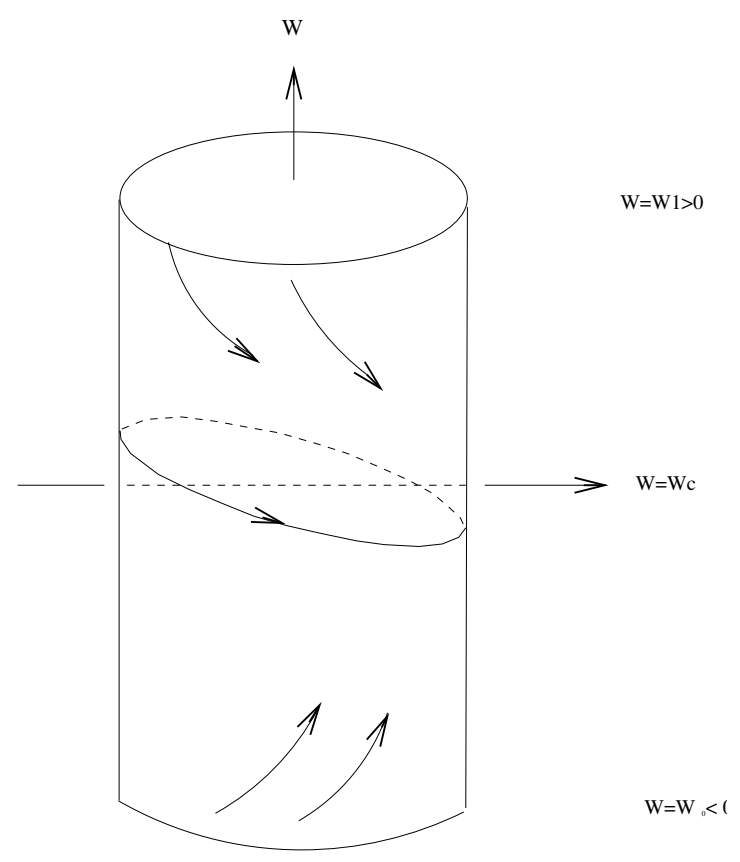

Figure 1. The limit cycle.

\subsection{An example}

Before starting with the example, we shall explain what should be a (weak) asymptotic optimal synthesis in the general Martinet case ( $\kappa \neq 0$ above). Starting with $(20)$, we consider the cylinder $C_{\varepsilon}$. Since $\Gamma$ is transversal to $\Delta$, and $\Gamma$ is compact, then for $\varepsilon$ small enough, as in the Section $4, \Delta$ is transversal also to $C_{\varepsilon}$, and therefore, $\Delta$ defines a vector field $X_{\varepsilon}$ on $C_{\varepsilon}$, (see Fig. 1).

We see in equation (20) that, for $|w|$ large, this vector field points in the direction of $w=0$. Therefore, by the Poincaré-Bendixon theorem, there is a limit-cycle.

It is possible to check that, for generic problems, this limit-cycle is not horizontal (not contained in a plane $\{w=$ constant $\}$ ), has size $\varepsilon^{3}$, and is centered at a point $w_{c}$ of order $\varepsilon^{2}$.

Then, a well defined synthesis goes under the following steps:

- starting from $w_{0}$, follow any integral curve of $X_{\varepsilon}$, up to the first time it crosses the plane $\left\{w=w_{c}\right\}$;

- following horizontal geodesic, use at most $2 \varepsilon$ units of time, to cross the plane $\left\{w=w_{c}\right\}$;

- following (in reversed time) a suitably chosen trajectory of $X_{\varepsilon}$, reach the desired destination $w_{1}$.

As we shall show in the example, this strategy gives a complexity which is strongly equivalent to $-\frac{12}{\alpha}$.

Example 5.2. Consider in $\mathbb{R}^{3}$ the frame

$$
\begin{aligned}
F & =\frac{\partial}{\partial x}+\frac{y}{2} \gamma \frac{\partial}{\partial w} \\
G & =\frac{\partial}{\partial y}-\frac{x}{2} \gamma \frac{\partial}{\partial w}
\end{aligned}
$$

with $\gamma=-\alpha w+a x$. 
The fact that $a$ is nonzero (an invariant fact in the general normal form) makes the distribution non-integrable, and the limit-cycle non horizontal. In cylindrical coordinates, $(w, \theta, \varepsilon)$, it is to easy to see that:

$$
X_{\varepsilon}=-\frac{1}{\varepsilon} \frac{\partial}{\partial \theta}+\frac{\varepsilon}{2}(-\alpha w+a \varepsilon \cos \theta) \frac{\partial}{\partial w}
$$

Then, we can explicitly compute the flow of $X_{\varepsilon}$, to obtain

$$
\begin{aligned}
\theta(t) & =-\frac{t}{\varepsilon}+\theta_{0}, \quad \text { and } \\
w(t) & =\mathrm{e}^{-\frac{\varepsilon \alpha}{2} t} w_{0}+\frac{\varepsilon^{2} a}{2} \int_{0}^{t} \mathrm{e}^{-\frac{\varepsilon \alpha}{2}(t-s)} \cos \theta(s) \mathrm{d} s .
\end{aligned}
$$

After straightforward computations, we obtain

$$
\begin{aligned}
w(t)= & \left(4+\alpha^{2} \varepsilon^{4}\right)^{-1}\left(\mathrm { e } ^ { - \frac { \varepsilon \alpha t } { 2 } } \left[4 w_{0}+\alpha^{2} \varepsilon^{4} w_{0}+a \alpha \mathrm{e}^{\frac{\varepsilon \alpha t}{2}} \varepsilon^{5} \cos \left(\frac{t}{\varepsilon}-\theta_{0}\right)\right.\right. \\
& \left.\left.\left.-a \alpha \varepsilon^{5} \cos \theta_{0}+2 a \mathrm{e}^{\frac{\alpha \varepsilon t}{2}} \varepsilon^{3} \sin \left(\frac{t}{\varepsilon}-\theta_{0}\right)+2 a \varepsilon^{3} \sin \theta_{0}\right)\right]\right)
\end{aligned}
$$

The expression for the limit cycle is the following

$$
\begin{aligned}
\theta(t) & =-\frac{t}{\varepsilon} \\
w(t) & =\frac{a \varepsilon^{3}}{4+\alpha^{2} \varepsilon^{4}}\left(\alpha \varepsilon^{2} \cos \left(\frac{t}{\varepsilon}\right)+2 \sin \left(\frac{t}{\varepsilon}\right)\right)
\end{aligned}
$$

which shows that $w_{c}=0$, and the size of the limit cycle is $\frac{a}{2} \varepsilon^{3}$.

Now, starting from $w_{0}>0$, we want to go to $w_{c}=0$, using (23). We get

$$
0=\mathrm{e}^{-\frac{\varepsilon \alpha}{2} t}\left(4 w_{0}+2 a \varepsilon^{3}\left(\sin \theta_{0}+\mathrm{e}^{\frac{\varepsilon \alpha}{2} t} \sin \left(\frac{t}{\varepsilon}-\theta_{0}\right)\right)\right)+O\left(\varepsilon^{4}\right)
$$

(where $\frac{O\left(\varepsilon^{4}\right)}{\varepsilon^{4}}$ is bounded). We rewrite this:

$$
4 w_{0} \mathrm{e}^{-\frac{\varepsilon \alpha}{2} t}=-2 a \varepsilon^{3}\left(\sin \theta_{0} \mathrm{e}^{-\frac{\varepsilon \alpha}{2} t}+\sin \left(\frac{t}{\varepsilon}-\theta_{0}\right)\right)+O\left(\varepsilon^{4}\right) .
$$

It is clear that this equation in $t$ has a solution, for which:

$$
\mathrm{e}^{-\frac{\varepsilon \alpha}{2} t} \simeq \frac{a}{2 w_{0}} \varepsilon^{3}
$$

Hence,

$$
t \stackrel{s}{\simeq}-\frac{6}{\alpha \varepsilon} \ln \varepsilon
$$

Taking into account the part $w(t)<0$ of the trajectory, we get that the complexity $C(\varepsilon)$ of our synthesis (complexity $=\frac{1}{\varepsilon}$ length):

$$
C(\varepsilon) \simeq-12 \frac{\ln \varepsilon}{\alpha \varepsilon^{2}}
$$




\section{The nOn Generic CASE}

\subsection{Normal form}

Here we shall restrict ourselves to $C^{\omega}$ (analytic) motion-planning problems. In this case, prenormal coordinates of Theorem 3.1, Section 3 still exist, and they are $C^{\omega}$ coordinates along the curve $\Gamma$.

Now, to obtain normal coordinates, (Th. 3.2, Sect. 3), we need the analog of Lemma 7.1, Appendix 7, for the analytic case. This is Lemma 7.2 of the same appendix, which claims that in fact, the smooth diagonalization property for the fundamental form $\widetilde{\alpha}$ always holds. Because of that, we have the following theorem:

Theorem 6.1 (normal Coordinates in the analytic case). Let $\Sigma=(F, G) \in S_{R}^{\omega}$. Then, without any extra assumption, the conclusions of Theorem 3.2, Section 3, still hold, and the normal coordinate system is compatible with the analytic structure.

We have also the analog of the Theorem 3.4, Section 3.2 in the analytic context. Let us repeat it precisely:

Theorem 6.2 (analytic normal form). Let $\Sigma=(F, \Gamma) \in S_{R}^{\omega}$, and let $(\xi, w)$ be a fixed normal $C^{\omega}$ coordinate system along the curve $\Gamma$ (it does exist by Th. 6.1). Then, there is a family of analytic functions $\alpha_{1}(\cdot), \ldots, \alpha_{n}(\cdot)$ : $[0,1] \longrightarrow \mathbb{R}$, such that the fundamental form $\widetilde{\alpha}$ along $\Gamma$ is

$$
\widetilde{\alpha}(w)=\alpha_{1}(w) \mathrm{d} x_{1} \wedge \mathrm{d} y_{1}+\cdots+\alpha_{n}(w) \mathrm{d} x_{n} \wedge \mathrm{d} y_{n}, \quad N=2 n+1, \text { or } 2 n+2 .
$$

Also, there is an orthonormal analytic sub-Riemannian frame $\mathcal{F}=(Q, L)$, with the same properties 1) to 7) as those of Theorem 3.4, Section 3.

The only difference is that these analytic functions $\alpha_{1}(\cdot), \ldots, \alpha_{n}(\cdot)$ are not necessarily positive everywhere and their graphs might cross each other.

\subsection{Metric complexity}

Let us make just the following assumption

$$
\kappa(w)=\sup _{i}\left|\alpha_{i}(w)\right| \neq 0 \quad \text { for all } \quad w \in[0,1] .
$$

In fact this is equivalent to say that the fundamental form $\widetilde{\alpha}(w) \neq 0$, for all $w \in[0,1]$. Then, the statements of Theorem 1.8 hold, namely

Complexity:

- under assumption (26), formula (2) for the metric complexity is still valid;

- the function $T \mapsto 2 \int_{0}^{T} \frac{\mathrm{d} t}{\kappa(t)}$ is of class $C^{1}$, piecewise analytic, and its second derivative has jumps at a finite number of times.

\subsection{Asymptotic optimal synthesis}

We describe now the corresponding asymptotic optimal synthesis for the non-generic case. The function $\kappa:[0,1] \rightarrow \mathbb{R}$ is clearly continuous, piecewise analytic, positive, with jumps on its derivative at a finite number of points, say $t_{1}, \ldots, t_{m}$. Between two successive times $t_{j}$ and $t_{j+1}$, the supremum in assumption (26) is reached by one of the eigenvalues moduli $\left|\alpha_{i_{j}}\right|$. Also between the consecutive times $t_{j}$ and $t_{j+1}$, we consider the cylinder $C_{\varepsilon}^{j}$ in the normal coordinates:

$$
C_{\varepsilon}^{j}=\left\{(\xi, w):\|\xi\|^{2}=\varepsilon^{2}, x_{i_{j}}^{2}+y_{i_{j}}^{2}=\varepsilon^{2}\right\} .
$$

For $\varepsilon$ small enough, $\Delta$ is transversal to $C_{\varepsilon}^{j}$, and defines a vector field $X_{\varepsilon}^{j}$ on $C_{\varepsilon}^{j}$. Asymptotic optimal synthesis:

- between $t_{j}$ and $t_{j+1}$ : follow the flow (any trajectory) of $X_{\varepsilon}^{j}$; 
- at $t=t_{j}$, or $t=0$, or $t=1$ : follow horizontal geodesics in the planes $\{w=$ constant $\}$, passing through $\Gamma$ :

- at $t=0$, in time exactly $\varepsilon$, reach $C_{\varepsilon}^{1}$ from $\Gamma(0)$;

- at $t=1$, in time exactly $\varepsilon$, go from $C_{\varepsilon}^{m}$ to $\Gamma(1)$;

- at $t=t_{j}$, join $C_{\varepsilon}^{j-1}$ to $C_{\varepsilon}^{j}$ in time $2 \varepsilon$.

The proof of the fact that this is a strong asymptotic optimal synthesis, and the proof of Theorem 1.8, are obvious modifications of the proof (Sect. 4) of Theorem 1.6: Theorem 6.2 allows us to repeat exactly the same arguments between two successive crossing points $t_{j}$ and $t_{j+1}$.

\section{Appendix}

\subsection{Diagonalization of 1-parameter families of skew-symmetric matrices}

Lemma 7.1. Let $A:[0,1] \rightarrow \mathfrak{s o}(N), t \mapsto A(t)$, be a smooth $\left(C^{\infty}\right)$ curve of real skew-symmetric matrices satisfying that for each $t \in[0,1]$ :

a) the kernel of $A(t)$ is at most 2-dimensional, and

b) $A(t)$ has no double nonzero eigenvalue.

Then, $A(t)$ is $2 \times 2$ block-diagonalizable by a smooth curve

$$
[0,1] \longrightarrow \mathrm{SO}(N), \quad t \longmapsto U(t)
$$

In the case $N=2 n+1$, there is an extra $1 \times 1$ block.

Proof. First, let us consider the map $[0,1] \rightarrow P_{N}(\mathbb{R}), t \mapsto A^{2}(t)$, where $P_{N}(\mathbb{R})$ denotes the space of real $N \times N$ symmetric matrices. By the assumptions a) and b), we have that for all $t \in[0,1]$, the eigenvalues of $A^{2}(t)$ are double (at most one is identically zero and it is simple, if $N=2 n+1$ ).

We take $P_{A(t)}(\lambda)$ to be either the characteristic polynomial of $A(t)$, if $N=2 n$, or this polynomial divided by $\lambda$, if $N=2 n+1$. Then, $P_{A(t)}(\lambda)$ is a polynomial of degree $n$ in $X=\lambda^{2}$.

All the roots of polynomial $P_{A(t)}(X)$ are simple. Hence, for a root $X_{0}$, we can take a $t_{0} \in[0,1]$, such that in a neighborhood of $t_{0}$, there is a smooth real-valued function $t \mapsto X(t)$ such that $X\left(t_{0}\right)=X_{0}$.

If $X_{0}<0$, then, in a neighborhood of $t_{0}$ we have that $\pm i \sqrt{-X(t)}$, is a smooth couple of distinct eigenvalue of $\mathrm{A}(\mathrm{t})$. The only thing that might be unclear is that if $X_{0}=0$ then $\pm i \sqrt{-X(t)}$ is smooth. In any case, we have at least $n-1$ positive smooth functions

$$
[0,1] \longrightarrow \mathbb{R}, t \longmapsto \alpha_{j}(t)
$$

such that $\pm i \alpha_{j}(t)$ are simple eigenvalues of $A(t)$. These functions are smooth and globally defined on [0,1]. Now fix one of these couples of eigenvalues $\pm i \alpha_{j}(t)$ and consider the maps:

$$
[0,1] \longrightarrow P_{N}(\mathbb{R}), t \longmapsto A^{2}(t)+\alpha_{j}^{2}(t) I d
$$

The kernels $V_{t}$ of these matrices $A^{2}(t)+\alpha_{j}^{2}(t) I d$, are well defined, smooth and 2-dimensional. Then the set of the couples $\left(t, V_{t}\right)$ defines a smooth sub-bundle of the trivial bundle

$$
\Pi:[0,1] \times \mathbb{R}^{N} \rightarrow[0,1]
$$

This sub-bundle is a vector bundle of 2-planes over [0,1]. Then, it is trivial and possesses a smooth nonzero section $t \rightarrow X_{t}$. We can assume that $\left\|X_{t}\right\|=1$. The restriction of the Euclidean metric $g$ on $\mathbb{R}^{N}$ to this bundle defines the following two smooth curves:

$$
t \longmapsto \pm Y_{t}, \quad \text { such that }\left\|Y_{t}\right\|=1,\left\langle X_{t}, Y_{t}\right\rangle_{g}=0 \text {. }
$$


We choose one of these curves, say $t \mapsto+Y_{t}$, and then, we have a smooth map $t \mapsto(X(t), Y(t))$, defined on $[0,1]$, such that for all $t \in[0,1]$, the couple $\left(X_{t}, Y_{t}\right)$ forms an orthonormal basis of the kernel:

$$
\operatorname{ker}\left(A^{2}(t)+\alpha_{j}^{2}(t) I d\right) .
$$

For $N=2 n+1$, we have also the mapping $t \mapsto \bar{V}_{t}=\operatorname{ker} A^{2}(t)$, which defines a bundle of lines over [0,1], that has also a nonzero smooth section $t \rightarrow \bar{X}_{t}$, with $\left\|\bar{X}_{t}\right\|=1$.

Now if $N=2 n$, it remains a single couple of eigenvalues, $\pm i \alpha_{n}(t)$ such that $\alpha_{n}^{2}(t)$ is smooth, but vanishes somewhere, and it is not clear that $\alpha_{n}(t)$ is smooth. But then the space:

$$
V_{t}=\operatorname{ker}\left(A^{2}(t)+\alpha_{n}^{2}(t) I d\right),
$$

is still well defined and smooth (we may also get it as the one which is orthogonal to all the other smooth vector bundles).

Then, in the same way, we obtain a couple $\left(X_{t}, Y_{t}\right)$ of smooth orthogonal vectors in this kernel. Now we have the mapping:

$$
[0,1] \longrightarrow \mathrm{SO}(N), \quad t \longmapsto U(t),
$$

which is smooth, and block-diagonalizes the curve $\mathrm{A}(\mathrm{t})$. This shows moreover that $t \mapsto \alpha_{n}(t)$ is smooth.

In the analytic case, there is a much stronger result. Let us consider the real $C^{\omega}$ map:

$$
[0,1] \longrightarrow \mathfrak{s o}(N), \quad t \longmapsto A(t) .
$$

It follows from known results in perturbation theory for linear operators, see for instance [13], that in the complex plane $\mathbb{C}$, there is a neighborhood $V([0,1]) \subset \mathbb{C}$ of the interval $[0,1]$, and a holomorphic map,

$$
V([0,1]) \longrightarrow M_{N}(\mathbb{C}), \quad t \longmapsto U(t),
$$

such that $U(t)$ is unitary when restricted to $[0,1]$, and diagonalizes $A(t)$ for all $t$. The following lemma is an immediate consequence of these considerations.

Lemma 7.2. Given any $C^{\omega}$ map

There is a $C^{\omega}$ map

$$
[0,1] \longrightarrow \mathfrak{s o}(N), \quad t \longmapsto A(t) .
$$

$$
[0,1] \longrightarrow \mathrm{SO}(N), \quad t \longmapsto U(t),
$$

that $2 \times 2$ block-diagonalizes $A(t)$ for all $t$. If $N=2 n+1$, then there is an extra $1 \times 1$ block.

\subsection{Distinguished sets of the Lie algebra of skew-symmetric matrices}

We consider in this paragraph basic properties of certain distinguished sets of the Lie algebra $\mathfrak{s o}(N)$ of skewsymmetric matrices, such sets play a role in the proofs of our results. First it is easy to prove the following general fact:

$$
\begin{aligned}
& \text { the subset } \widehat{\mathcal{A}}_{n} \subset \mathfrak{s o}(N) \text { of matrices with kernel of dimension exactly } n \text {, is a sub-manifold of } \\
& \text { codimension } \frac{n(n-1)}{2} \text {. }
\end{aligned}
$$

Observe that not all $n$ are possible for each $N$, since both must have the same parity. As easy consequences of this general result we have the following three properties.

$F_{1}$. The subset $\mathcal{A}_{1}$ of $\mathfrak{s o}(2 n)$ of matrices that have a nonzero kernel, is an algebraic closed set with codimension 1.

$F_{2}$. The subset $\mathcal{A}_{3}$ of $\mathfrak{s o}(2 n+1)$ of matrices that have a non-minimum kernel (i.e. not of dimension 1$)$ is an algebraic closed set with codimension 3 . 
$F_{3}$. The subset $\mathcal{A}_{2}$ of $\mathfrak{s o}(2 n)$ of matrices that have a kernel of codimension $>2($ i.e. $\geq 4)$ is algebraic, closed, and of codimension 6 .

The following property is less straightforward and we shall provide a proof.

$F_{4}$. The subset $\mathcal{A}_{4}$ of $\mathfrak{s o}(N)$ of matrices with a double nonzero eigenvalue is semi-algebraic, of codimension 3 . Its closure $\overline{\mathcal{A}}_{4}$ has the same codimension.

Proof. The fact that $\mathcal{A}_{4}$ is semi-algebraic can be easily verified, we check only that the codimension of $\mathcal{A}_{4}$ is $\geq 3$. In fact, this is the only result we use in the paper. The set $\mathcal{A}_{4}$ is the union of the orbits under conjugation in $\mathrm{SO}(N)$ of block-diagonal matrices of the form:

$$
\begin{cases}\operatorname{diag}\left(\alpha J, \alpha J, \alpha_{3} J, \ldots, \alpha_{n} J\right), & \text { if } N=2 n, \text { and } \\ \operatorname{diag}\left(\alpha J, \alpha J, \alpha_{3} J, \ldots, \alpha_{n} J, 0\right), & \text { if } N=2 n+1\end{cases}
$$

with $\alpha \neq 0$ and $J=\left(\begin{array}{cc}0 & -1 \\ 1 & 0\end{array}\right)$.

Let $\mathcal{D}$ be the set of such matrices, and let $\widehat{\mathcal{A}_{4}} \subset \mathcal{D}$ be the set of generic elements of $\mathcal{D}$, that is, those elements for which the matrices

$$
\begin{cases}\operatorname{diag}\left(\alpha_{3} J, \ldots, \alpha_{n} J\right), & \text { if } N=2 n, \quad \text { and } \\ \operatorname{diag}\left(\alpha_{3} J, \ldots, \alpha_{n} J, 0\right), & \text { if } N=2 n+1,\end{cases}
$$

are regular elements of $\mathfrak{s o}(N-4)$, that further satisfy $\alpha \neq 0, \alpha \neq \pm \alpha_{j}$ and $\alpha_{j} \neq 0$.

Then, for $D \in \widehat{\mathcal{A}_{4}}$ an easy computation shows that ker $a d D=\mathfrak{g} \times \mathfrak{c}_{N-4}$, where $\rfloor_{N-4}$ is a Cartan sub-algebra of $\mathfrak{s o}(N-4)$, and $\}$ is the Lie algebra of matrices of the form:

$$
\left(\begin{array}{rrrr}
0 & a & c & d \\
-a & 0 & -d & c \\
-c & d & 0 & e \\
-d & -c & -e & 0
\end{array}\right)
$$

Let now $G$ be the 4-dimensional Lie group corresponding to $\mathfrak{g}$. The stabilizer $S_{1}$ of $D \in \widehat{\mathcal{A}_{4}}$ is $S_{1}=\mathrm{T}(N-4) \times G$, where $\mathrm{T}(N-4)$ is a maximal torus of $\operatorname{SO}(N-4)$. Its dimension is $\operatorname{dim}\left(S_{1}\right)=4+n-2=n+2$. Therefore, the orbit under conjugation of $D$ has dimension $M-n-2$, where

$$
M=\frac{N(N-1)}{2}
$$

Stabilizers of non-generic points of $\mathcal{D}$ are bigger, hence the orbits of these points have lower dimension. At the end, all of these orbits under conjugation of the elements of $\mathcal{D}$ have dimension $\leq M-n-2$. Since the dimension of $\mathcal{D}$ is $n-1$, it follows that the dimension of $\mathcal{A}_{4}$ is smaller than $M-n-2+n-1=M-3$. Hence:

$$
\operatorname{codim} \mathcal{A}_{4} \geq 3
$$

Acknowledgements. The authors want to thank Frédéric Jean for the fruitful discussions and for his thorough observations about our results.

The present paper was written while the third author (Monroy-Pérez) was on sabbatical leave at INRIA, Unité de Recherche Sophia-Antipolis, France. The author thanks Prof. J.B. Pomet and the rest of the team MIAOU for their support. He was also partly supported by the CONACYT's grant for sabbatical periods abroad. 


\section{REFERENCES}

[1] R. Abraham and J. Robbin, Transversal mappings and flows. W.A. Benjamin, Inc. (1967).

[2] A. Agrachev, El-A. Chakir, El-H. and J.P. Gauthier, Sub-Riemannian metrics on $R^{3}$, in Geometric Control and nonholonomic mechanics, Mexico City (1996) 29-76, Canad. Math. Soc. Conf. Proc. 25, Amer. Math. Soc., Providence, RI (1998).

[3] A. Agrachev and J.P. Gauthier, Sub-Riemannian Metrics and Isoperimetric Problems in the Contact case, L.S. Pontriaguine, 90th Birthday Commemoration, Contemporary Mathematics 64 (1999) 5-48 (Russian). English version: J. Math. Sci. 103, 639-663.

[4] M.W. Hirsch, Differential Topology. Springer-Verlag (1976).

[5] El-A. Chakir, El-H., J.P. Gauthier and I. Kupka, Small Sub-Riemannian balls on $R^{3}$. J. Dynam. Control Syst. 2 (1996) 359-421.

[6] G. Charlot, Quasi-Contact sub-Riemannian Metrics, Normal Form in $R^{2 n}$, Wave front and Caustic in $R^{4}$. Acta Appl. Math. 74 (2002) 217-263.

[7] K. Goldberg, D. Halperin, J.C. Latombe and R. Wilson, Algorithmic foundations of robotics. AK Peters, Wellesley, Mass. (1995).

[8] Mc Pherson Goreski, Stratified Morse Theory. Springer-Verlag, New York (1988).

[9] M. Gromov, Carnot-Caratheodory spaces seen from within, in Sub-Riemannian geometry. A. Bellaiche, J.J. Risler Eds., Birkhauser (1996) 79-323.

[10] F. Jean, Complexity of nonholonomic motion planning. Internat. J. Control 74 (2001) 776-782.

[11] F. Jean, Entropy and Complexity of a Path in Sub-Riemannian Geometry. ESAIM: COCV 9 (2003) 485-508.

[12] F. Jean and E. Falbel, Measures and transverse paths in Sub-Riemannian Geometry. J. Anal. Math. 91 (2003) 231-246.

[13] T. Kato, Perturbation theory for linear operators. Springer-Verlag (1966) 120-122.

[14] I. Kupka, Géometrie sous-Riemannienne, in Séminaire Bourbaki, 48 année, No. 817 (1995-96) 1-30.

[15] G. Lafferiere and H. Sussmann, Motion Planning for controllable systems without drift, in Proc. of the 1991 IEEE Int. Conf. on Robotics and Automation (1991). 